\title{
Sixth Generation (6G) Cognitive Radio Network (CRN) Application, Requirements, Security Issues, and Key Challenges
}

\author{
Muhammad Muzamil Aslam $\mathbb{D D}^{1,2,3}$ Liping Du ${ }^{1 D},{ }^{2,4}$ Xiaoyan Zhang $\mathbb{D}^{5},{ }^{5}$ Yueyun Chen $\mathbb{D}^{2}{ }^{2}$ \\ Zahoor Ahmed $\mathbb{D}^{6,7}$ and Bushra Qureshi ${ }^{1}{ }^{1}$ \\ ${ }^{1}$ Department of Electronics and Communication Engineering, University of Science and Technology China (USTC), Hefei, \\ Anhui 230026, China \\ ${ }^{2}$ School of Computer \& Communication Engineering, University of Science and Technology Beijing, Beijing 100083, China \\ ${ }^{3}$ Department of Information Sciences, University of Education (UoE), Multan, Pakistan \\ ${ }^{4}$ Shunde Graduate School of University of Science and Technology Beijing, Beijing 100083, China \\ ${ }^{5}$ State Radio Monitoring Center, Beijing 100037, China \\ ${ }^{6}$ Department of Automation, Shanghai Jiaotong University, Shanghai 200240, China \\ ${ }^{7}$ Department of Electronics, Government College University (GCU) Lahore, Pakistan
}

Correspondence should be addressed to Liping Du; dlp2001@ies.ustb.edu.cn and Zahoor Ahmed; zahoorgcu10@sjtu.edu.cn

Received 28 July 2021; Revised 13 September 2021; Accepted 29 September 2021; Published 13 October 2021

Academic Editor: VIMAL SHANMUGANATHAN

Copyright (c) 2021 Muhammad Muzamil Aslam et al. This is an open access article distributed under the Creative Commons Attribution License, which permits unrestricted use, distribution, and reproduction in any medium, provided the original work is properly cited.

Recently, 5G installation has been started globally. Different capabilities are in the consistent procedure, like ultrareliability, mass connectivity, and specific low latency. Though, $5 \mathrm{G}$ is insufficient to meet all the necessities of the future technology in 2030 and so on. Next generation information and communication technology is playing an important role in attraction of researchers, industries, and technical people. With respect to $5 \mathrm{G}$ networks, sixth-generation $(6 \mathrm{G}) \mathrm{CR}$ networks are anticipated to familiarize innovative use cases and performance metrics, such as to offer worldwide coverage, cost efficiency, enhanced spectral, energy improved intelligence, and safety. To reach such requirements, upcoming $6 \mathrm{G}$ CRNs will trust novel empowering technologies. Innovative network architecture and transmission technologies and air interface are of excessive position, like multiple accesses, waveform design, multiantenna technologies, and channel coding schemes. (1) To content, the condition should be of worldwide coverage, there will be no limit on $6 \mathrm{G}$ to global $\mathrm{CR}$ communication networks that may require to be completed with broadcast networks, like satellite communication networks, therefore, attaining a sea integrated communication network. (2) The spectrums overall will be entirely travelled to the supplementary rise connection density data rates in optical frequency bands, millimeter wave (mmWave), sub-6 GHz, and terahertz (THz). (3) To see big datasets created because of tremendously varied CR communication networks, antenna rush, diverse communication scenarios, new provision necessities, wide bandwidth, and 6G CRNs will allow an innovative variety of intelligent applications with the assistance of big data and AI technologies. (4) Need to improve network security when deploying 6G technology in CR networks. 6G is decentralized, intended, intelligent innovative, and distributed network. In this article, we studied a survey of current developments and upcoming trends. We studied the predicted applications, possible technologies, and security issues for $6 \mathrm{G}$ CR network communication. We also discussed predicted future key challenges in $6 \mathrm{G}$.

\section{Introduction}

From 2020, 5G cognitive radio networks (CRNs) [1] are playing a key role in global communication and spectrum sharing. The main part of $5 \mathrm{G}$ CR communication is massive machinetype communication, enhanced mobile broadband, and low latency ultrareliable communication. The main features of $5 \mathrm{G}$ are about $0.1 \mathrm{Gbps}$ data rate, the peak data rate is about $20 \mathrm{Gbps}$, end-to-end latency is about $1 \mathrm{~ms}$, device connectivity is approximately $1 \mathrm{million} / \mathrm{km}^{2}$, thrice spectrum efficiency, $500 \mathrm{~km} / \mathrm{h}$ supporting mobility, traffic capacity by area $10 \mathrm{Mbps} / \mathrm{m}^{2}$, and several times energy efficiency as compared 
to $4 \mathrm{G}$ CRN communication [2]. Several essential technologies, such as ultradense network, millimeter-wave, and MIMO, have been studied to gain $5 \mathrm{G} \mathrm{CRN} \mathrm{communication.}$

Although, fifth-generation is insufficient to meet the necessities of 2030 and so on. Scientists, institutes, and researchers focus on $6 \mathrm{G} \mathrm{CRN}$ communication $[3,4]$. An essential point of fifth-generation $(5 G)$ is low latency, which requires a deterministic network to ensure point-to-point inactivity with the requirement and precision of incoming future mandate. Authors studied in [5] that $6 \mathrm{G}$ must be capable to pursue high synchronization than 5G. Furthermore, there will be about maximum geographical coverage, millisecond geographical location updating accuracy, and subcentimeter geographical accuracy in 6G [6].

In some cases, 5G is still complicated, e.g., villages, remote areas, and roads are not fully covered by $5 \mathrm{G}$ that confines few applications, like driverless vehicles. Specific communication satellite and nonterrestrial networks must fulfill broadcast networks for ubiquitous services surety, seamless, and cost-effectiveness. The communication network of the unmanned aerial vehicle is essential for quick reply in any complication of surroundings. Although millimeter-wave can offer Gbps data transmission in 5G, it will require a Tbps data rate in $6 \mathrm{G}$ for applications $[7,8]$ such as $3 \mathrm{D}$ high-quality videos, virtual reality, augmented reality, alternative frequency bands, and $\mathrm{THz}$ may be contender bands $[9,10]$. With the use of extraordinary heterogeneous networks, number of antennas in large amount, new service diverse communication situation, and wide bandwidth, there will be new access of smart applications in 6G with the assistance of machine learning $[11,12]$ and artificial intelligence technologies. There is an automation level for network performance improvement in several forms, such as quality of security, quality of services, fault management, experience quality, and energy efficiency [13-15].

In $5 \mathrm{G}$, network traffic is dominated by streaming or video applications. Except for all the applications mentioned above and requirements, we can study from $5 \mathrm{G}$ physical information communication $[16,17]$ which CR networks are robotics controlled. Factory logistics and automated driving are fresh cellular technology applications, but still, there are some new challenges. Using such applications for network traffic analysis, several mobile objects transfer control and sensor information overload the central network in control system. Artificial intelligence used distributed control systems is developing research focus for future technology and demand. It is to be supposed that artificial intelligence and such application networks will dominate CR network traffic requirement of $6 \mathrm{G}$. This area of research is untouched that makes its presence challenging [18].

To see 5G CR networks, 6G CR networks will provide maximum higher spectral, cost efficiency, energy, maximum data rate about in Tbps, about ten-time lower latency, connection density about 100 times improved, and maximum intelligence for automation. Latest transmission and spectrum sensing technologies and air interference are mandatory to gain energy efficiency and high spectrum efficiency with fresh waveforms, channel coding methods, multiple access approaches, and multiantenna technologies are the proper combination of all such techniques. Meanwhile, such network architecture requires dynamic network slicing, software-defined network, cognitive service architecture, service-based architecture, and free of cell architecture. Although, the software comes at a point as we acknowledged from $5 \mathrm{G}$ progress. A commercial use server versus domain particular chips in the virtualized radio access network (VRAN) suggests a massive amount of energy consumption enhancement and measure to better energy efficiency. The current statistic shows that power consumption in $5 \mathrm{G}$ is more than $4 \mathrm{G}$ networks but at higher bandwidth.

Contrary to this, we should provide a network that is stimulatingly of its start does not cross the limits of previous generation power requirement. So, in $6 \mathrm{G}$, we need a new computing paradigm to provide all advantages of notarization deprived of energy consumption cost. Some computing technologies like fog computing, edge computing, and cloud computing are key in network flexibility, processing, lower latency, synchronization, and distributed computing. To overcome the limitations and drawback of $5 \mathrm{G}$ short packet, the network should provide low latency service using high data rate, delivery of high reliability, internet of everything, and system coverage. To overcome the requirements of CRN communication in 2030 and beyond [19], 6G CR network should develop the data rate or human-centric [20]. To acquire such supplies, 6G CR network communication will shift to a new paradigm.

We will illustrate the $6 \mathrm{G}$ CR network vision in this paper. The first $6 \mathrm{G} \mathrm{CR}$ networks will be sea integrated space air-ground networks for global network communication coverage. UAV communication, satellite communication, and naval communication will encompass vast areas for CR network communication coverage. All spectrums will be evident for providing a high data rate. For full application enabling, machine learning and artificial intelligence technologies will be competently combined with 6G CR networks communication for better automation and network management [21]. Besides, artificial intelligence technology can enable dynamic arrangement of a network, resource computing for performance betterment, and caching of $6 \mathrm{G}$ networks. Last but not least, high network security is required for the physical and network layer during development. Industry verticals like the internet of things (IoT) [22, 23], virtual cloud reality, industry automation, digital body area network, cellular vehicle to everything, energy-efficient CR network, and central acknowledged system would boost the progress of $6 \mathrm{G} \mathrm{CR}$ network communication. $6 \mathrm{G} \mathrm{CR}$ network overview is shown in Figure 1. There is detailed intuition of $6 \mathrm{G}$ in Figure 2. Table 1 is for the acronyms used in the paper.

\section{Background, 6G Artificial Intelligence CR Network}

In this work, we focused that artificial intelligence that will empower 6G in all forms, network management and composition, signal processing and physical layer, service-based communication, and data mining [24]. Researchers have started planning and envisioning the essential technologies 


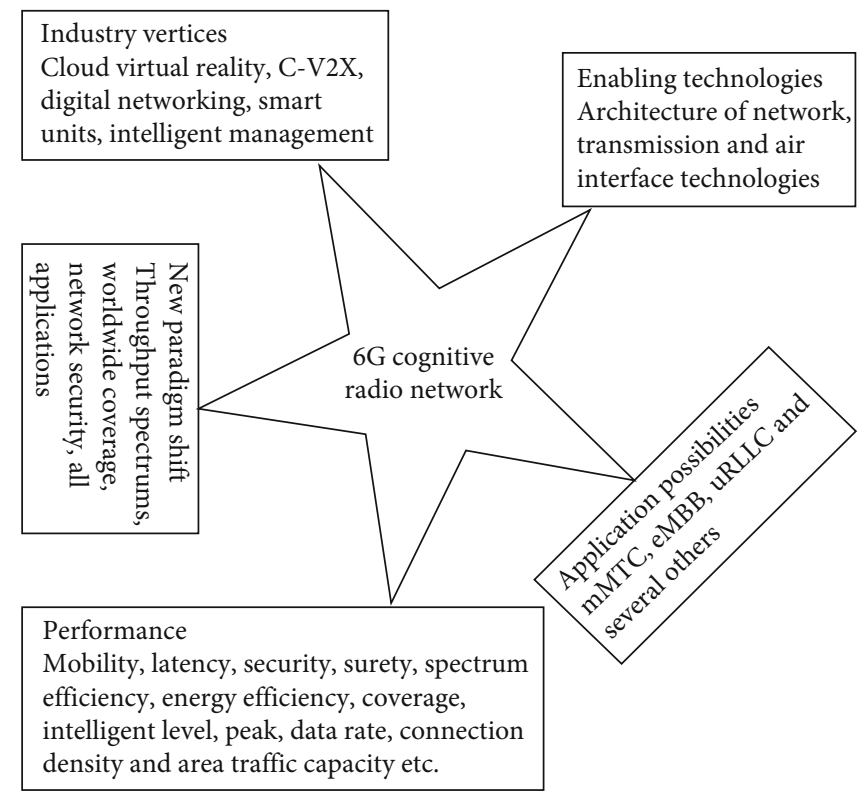

FIgURe 1: An overview on 6G cognitive radio networks.

supporting $6 \mathrm{G}$ CR network communication in various ways like beyond $5 \mathrm{G}+$. To gain $1 \mathrm{~ms}$ latency order, uRLLC was introduced in 5G for critical applications latency. Mobile edge computing and computing models defined in 5G for delay reduction and network overcrowding are classically met in user equipment to integrate base station CR network communication. Although, fog nodes are unable to act as freely relay and cloud data center on basic cloud. Another derivative of cloud computing is the cloud radio access network (CRAN), in which already present base stations are swapped with distributed remote radio heads and middle baseband unit (BBU). In the cloud radio access network architecture, signal processing computations are performed at the baseband unit. Although this cloud access radio network may complete the $5 \mathrm{G}$ requirements, service heterogeneously, communication overhead, and BBU performed computation, six generation $6 \mathrm{G}$ network will improve computational complexity and latency [25]. A progressing notation to focus on this is the open radio access network (O-RAN) that holds openness and intelligence as its key idea. O-RAN supplies clearly defined open boundaries between elements applied on universal purpose hardware and the integration of RRHs and BBUs from different retailers. Cell compaction is a primary enabler for accomplishing amplified network capacity [26]. 6G intentions at ultradense network distributions relating to multiple cells within a microcell. Diverse $6 \mathrm{G}$ use, such as high accuracy engineering, intelligent homes, and vehicle-to-everything, will involve various devices functioning in a microcell. Though, these 6G applications will include addressing numerous key challenges. The high-frequency operation, heterogeneity, mobility, and dense operation will present a different dimension of propagation, radio resource allocation, spectrum access, security concerns, and scheduling. Such sprints will be worsened with the intended 3D communication infrastructure [27] for 6G joining mobile ground and aerial platforms. Large-scale dense placement strategic for 6G, intelligent physical layer schemes will play a vital role in satisfying the provision requirements.

Furthermore, machine learning (ML) study at an advantage will become the leading enabler for $6 \mathrm{G}$ CR network communications. Deep learning (DL) explanations for CR network communication with MIMO beamforming, channel estimation and equalization, coding, error correction, signal recognition, etc., are computationally composite demanding controlling computational platforms. Though, DL architectures' proficient learning aptitude inspires the need to integrate them for future CR networks. Also, meaningful innovations in artificial intelligence have admonished it to be part of $5 \mathrm{G}$ yet organized only in services with substantial training data and commanding computing platforms. Though, to ease ML at an advantage, igniter operations of DL solutions will require to be established. Moreover, the black-box nature of neural networks reduces them unintelligible and erratic as it is challenging to increase vision into the educated function from network architecture.

Consequently, neural networks (NNs) present three main challenges: black-box nature, computational complexity, and resource constraint. Baseband tactile layer signal processing techniques will track the UE and base stations [28]. To see in a generation to generation of communication standard is bottlenecked by the UE's aptitude. Therefore, performing these DL explanations in the present UE stands will be impracticable as they do not own controlling computational platforms as obligatory by such tactics. Subsequently, intelligent baseband signal processing intended for 6G CR networks must be personalized for unimportant embedded computational daises with ruthless energy efficiency, latency objectives, and reliability. Combining domain information into the DL architectures embraces huge potential to rush the training process and model junction though attractive the model effectiveness. Table 2 is representing $5 \mathrm{G}$ to $6 \mathrm{G}$ evaluation.

\section{Cognitive Radio and Wireless Sensor Network (CR and WSN)}

With time, WSN used in IoT is increasing in the intelligent industry and almost covering all sectors of life such as e-health, traffic, smart homes, smart grids, intelligent stations, smart industry, monitoring purpose, and smart agriculture. WSN and CR can be in profit by sharing the process. Progressing installation of WSN can be oppressed by $\mathrm{CR}$ by $\mathrm{PU}$ channel monitoring, e.g., the new business model of spectrum sensing as a service (SSaaS). In another way, a large number of WSN usage increases the requirement of spectral resources. In this, CR is to be supposed as the solution to spectrum sharing.

3.1. For WSN Communication Dynamic Spectrum Sharing. Progressing the use of WSN devices requires improved use of limited frequency resources. For WSN, dynamic spectrum sharing WSN node is taken as SUs by CR. Keeping an eye on the PU channel, extra work is beard by WSN nodes, and good energy use requires SS task completion because energy 


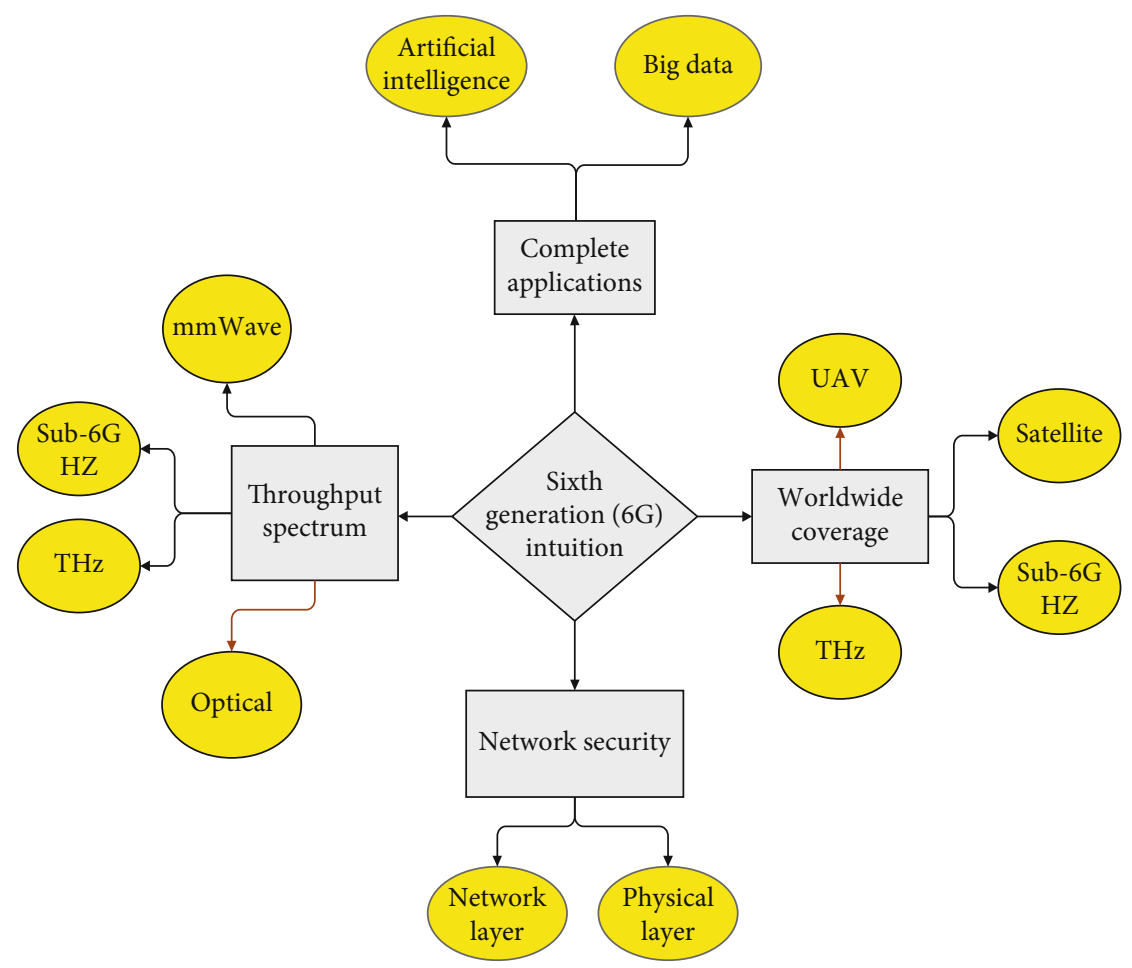

FIgURE 2: Sixth generation (6G) intuition.

consumption for WSN is vital since under protocols nodes are working for many years extension of a lifetime [29].

The CR procedure is complicated and challenging to WSNs because of many problems like spectrum sensing capability installation, sensing nodes with present frequency band, and node responsibility selection for nodes responsible for spectrum sensing.

CR's progressing technique is looking high requirement for frequency resource WSNs. Still, CR-WSN should give spectral efficiency and energy efficiency for industrial usage and applications. Transmission protocols and installation patterns play an essential role in reducing interference and lessing energy consumption between node assisting to improve the energy efficiency of CR-WSN networks. Although, different challenges have been seen as two ways exchange of data in suitable protocols, central object transmitting, and using SU data. The central object should be aware of the sensing channel SUs to be sensed and then give a decision response by these SUs. It executes frequency and time sync, such as the control channel. After detection of the present channel, transmission requires nodes or SUs should be aware by satisfactory channel for circuit configuration.

\section{Performance and Applications of CRN in 6G}

6G CR networks are projected to attain superior performance compared to $5 \mathrm{G}$ and possess high-performance matrices [30-32]. For 6G, the peak data rate is expected 1-10 Tbps with optical frequency band and $\mathrm{THz}$ assistance, while this data rate in $5 \mathrm{G}$ is $20 \mathrm{Gbps}$. User utilized data rate can gain a Gbps level with such maximum frequency bands. Traffic capacity by zone may be more than $1 \mathrm{Gbps} / \mathrm{m}^{2}$. There is the possibility to increase the spectrum efficiency from 3 to 5 times, and energy efficiency should also increase several times (about 100 times) compared to 5G. This is possible to attain by applying artificial intelligence to attain better network automation and management. The connection density will increase about a thousand times because of tremendously heterogeneous networks, huge antennas, wide bandwidth, and diverse communication situations. Mobility will be approximately $500 \mathrm{~km} / \mathrm{h}$ because of satellite movements, high-speed trains, and UAVs. The predicted latency for the selected set of applications is less than $1 \mathrm{~ms}$. Furthermore, some key matrices include security capacity, cost efficiency, intelligence level, and capacity. There is the primary support for human-centric CRNs communication notation in 2030 consisting of wearable device communication, implantable sensors, integrated headsets, smart industry 4.0, intelligent cars, human bonds communication, and robotics [33-35].

Furthermore, conventional CR users' devices such as laptops, mobile phones, and taking health-related intelligent devices into our consideration, frequency band and transmit power used in such devices should be under strict restrictions. Such devices will function on the human body and gather required data; security and weight of the device should be considered during the manufacturing and designing process. In 5G, about 256-1024 antennas realized massive MIMO. While comparing to 5G, 6G will deploy several times more antennas, in 6G, about 10000 antennas will be deployed such as ultramassive spatially massive MIMO where information is transmitted on few antennas by transmitter, and it decreases active antenna element. umMTC and feMBB are to offer massive connectivity, high data rates, and low latency. Figure 3 is showing an overview 
TABLE 1: Used abbreviations and explanation.

\begin{tabular}{|c|c|c|}
\hline Serial no. & Abbreviation & Explanation \\
\hline 1 & $4 \mathrm{G}$ & Fourth generation \\
\hline 2 & $5 \mathrm{G}$ & Fifth generation \\
\hline 3 & $6 \mathrm{G}$ & Sixth generation \\
\hline 4 & CRNs & Cognitive radio networks \\
\hline 5 & $\mathrm{CR}$ & Cognitive radio \\
\hline 6 & $\mathrm{THz}$ & Terahertz \\
\hline 7 & MIMO & Multiple-input multiple-output \\
\hline 8 & VRAN & Virtualized radio access network \\
\hline 9 & UAV & Unmanned aerial vehicle \\
\hline 10 & uRLLC & Ultrareliable low latency communication \\
\hline 11 & CRAN & Cloud radio access network \\
\hline 12 & $\mathrm{BBU}$ & Base band unit \\
\hline 13 & O-RAN & Open access radio network \\
\hline 14 & RRHS & Remote radio heads \\
\hline 15 & ML & Machine learning \\
\hline 16 & DL & Deep learning \\
\hline 17 & NNs & Neural networks \\
\hline 18 & UE & User equipment \\
\hline 19 & WSN & Wireless sensor network \\
\hline 20 & IoT & Internet of things \\
\hline 21 & PUs & Primary users \\
\hline 22 & SUs & Secondary users \\
\hline 23 & SSaaS & Spectrum sensing as a service \\
\hline 24 & $\mathrm{u} / \mathrm{mMTC}$ & Ultra/massive machine type communication \\
\hline 25 & $\mathrm{eMBB}$ & Enhanced mobile broadband \\
\hline 26 & feMBB & Further enhanced mobile broadband \\
\hline 27 & LDHMC & Long-distance and high-mobility communications \\
\hline 28 & ELPC & Extremely low-power communications \\
\hline 29 & uHSLLC & Ultrahigh speed with low latency communication \\
\hline 30 & uMUB & Ubiquitous mobile ultrabroadband \\
\hline 31 & uHDD & Ultrahigh data density \\
\hline 32 & ITU & International telecommunication union \\
\hline 33 & ITU-T & International telecommunication union-technology \\
\hline 34 & MR & Mixed reality \\
\hline 35 & $\mathrm{AR}$ & Augmented reality \\
\hline 36 & $\mathrm{XR}$ & Extended reality \\
\hline 37 & $\mathrm{M} 2 \mathrm{M}$ & Machine to machine \\
\hline 38 & $\mathrm{M} 2 \mathrm{H}$ & Machine to human \\
\hline 39 & $\mathrm{H} 2 \mathrm{M}$ & Human to machine \\
\hline 40 & $\mathrm{BCI}$ & Brain computer interface \\
\hline 41 & ORT & Optical radio technology \\
\hline 42 & $\mathrm{RF}$ & Radio frequency \\
\hline 43 & FSO & Free-space optical communication \\
\hline 44 & LiDAR & Light detection and ranging \\
\hline 45 & ITU-R & International telecommunication union-radio communication \\
\hline 46 & OF & Optical fiber \\
\hline 47 & LPWAN & Low-power wide-area network \\
\hline 48 & SRS & Smart reflecting surface \\
\hline
\end{tabular}


TABLE 1: Continued.

\begin{tabular}{lcc}
\hline Serial no. & Abbreviation & Explanation \\
\hline 49 & IoE & Internet of everything \\
50 & SS & Spectrum sensing \\
51 & QoS & Quality of service \\
52 & VR & Virtual reality \\
53 & CPS & Cyber physical systems \\
54 & E2E & Everything to everything \\
55 & AI & Artificial intelligence \\
\hline
\end{tabular}

TABLE 2: $6 \mathrm{G}$ evaluation in CR networks communication from $5 \mathrm{G}$ to $6 \mathrm{G}$.

\begin{tabular}{lcc}
\hline Problem & $5 \mathrm{G}$ & $6 \mathrm{G}$ \\
\hline Per device speed & $20 \mathrm{GBps}$ & 1 terabytes per second (Tbps) \\
Latency E2E & $10 \mathrm{~ms}$ & 1 millisecond (ms) \\
AI & Partial & Fully \\
XR & Partial & Fully \\
Mobility support & $500 \mathrm{~km} / \mathrm{hr}$ & 1000 kilometer per hour (km/hr) \\
Higher spectral efficiency & $30 \mathrm{bps} / \mathrm{HZ}$ & 100 bit per second/hertz (bps/HZ) \\
Haptic communication & Partial & Fully \\
Autonomous vehicle & Partial & Fully \\
Delay jitter & $\mathrm{NA} \mathrm{ms}$ & $10^{-3}$ millisecond (ms) \\
Energy efficiency & $\mathrm{NA}$ & 1 terabyte/joule (Tb/J) \\
Packet error rate & $10^{-5}$ & $10^{-9}$ \\
Higher channel bandwidth & $1 \mathrm{GHz}$ & 100 gigahertz $(\mathrm{GHz})$ \\
\hline
\end{tabular}

of $6 \mathrm{G}$ CRN communication. $5 \mathrm{G}$ focused on uRLLC, umMTC, and $\mathrm{eMBB}$, while $6 \mathrm{G}$ CRNs will widely improve also spread the application situations. There are three situations discussed umMTC, feMBB, and enhanced uRLLC in $[20,36]$. Many other application situations like ELPC and LDHMC are auspicious. Given three situations were called the ultrahigh speed with low latency communication (uHSLLC), ubiquitous mobile ultrabroadband (uMUB), and ultrahigh data density (uHDD) in [37]. Authors in [38] also studied several other applications and situations like the mobile ultrawideband, super IoT, and AI. International Telecommunication Union (ITU) worked on initial research on 6G in February 2020. Its sixth-generation technology and dreams are predicted to accomplish in 2023 [27, 39]. Technology group of ITU focused for 2030 networks researched by ITU-T network group 13 at Geneva meeting 2018. It aims to research the aptitudes of networks for 2030 and beyond. In short, 6G applications of CR networks contain three $5 \mathrm{G}$ situations/scenarios and some new scenarios $[40,41]$. Some basic applications of $6 \mathrm{G} \mathrm{CR}$ networks are studied below. Figure 4 is showing possible predicted CR network communication [33, 42, 43].

4.1. Reality Extended. Reality extended or extended reality facilities containing mixed reality (MR), augmented reality $(A R)$, and virtual reality (VR) are key features of the $6 \mathrm{G}$ CR network communication system. Such features use 3D objects, and AI is the basic element of such features. In addition, they are providing perceptual needs of cognition, human sense, computing, physiology, and storage. 6G will avail guaranteed immersive VR/AR/MR understanding by joint high quality and designed integration of $6 \mathrm{G}$ CR network system. Live view of the physical world is possible by augmented reality (AR), that is, parts are amplified by different sensor inputs by way of visuals, video, audio, and worldwide aligning data system. Present reality uses and makes addition by some sort of devices. While computersimulated $3 \mathrm{D}$ experienced is virtual reality (VR), where computer technology uses authorized headsets to generate realistic feelings and imitate actual surroundings and create the unreal world. VR surroundings engage five human senses. In the end, MR merAR and VR generate a new atmosphere and visualization to attract in the actual period. Mixed reality (MR) is also called hybrid reality. Its key characteristics are fundamental, and artificial world contents can reply to each other in real-time. XR is a combination of natural and virtual surroundings and human-machine communication generated by wearable computer technology. It consists of all forms like VR, AR, and MR. XR brings these under a single term. Low latency, reliable, and high data rate CRN systems connectivity given in 6G system are significant for original XR experience [44].

4.2. Smart Housing Societies. The key role of $6 \mathrm{G}$ will rush life quality improvement, automation, and surrounding monitoring with the use of artificial intelligence-based machine- 


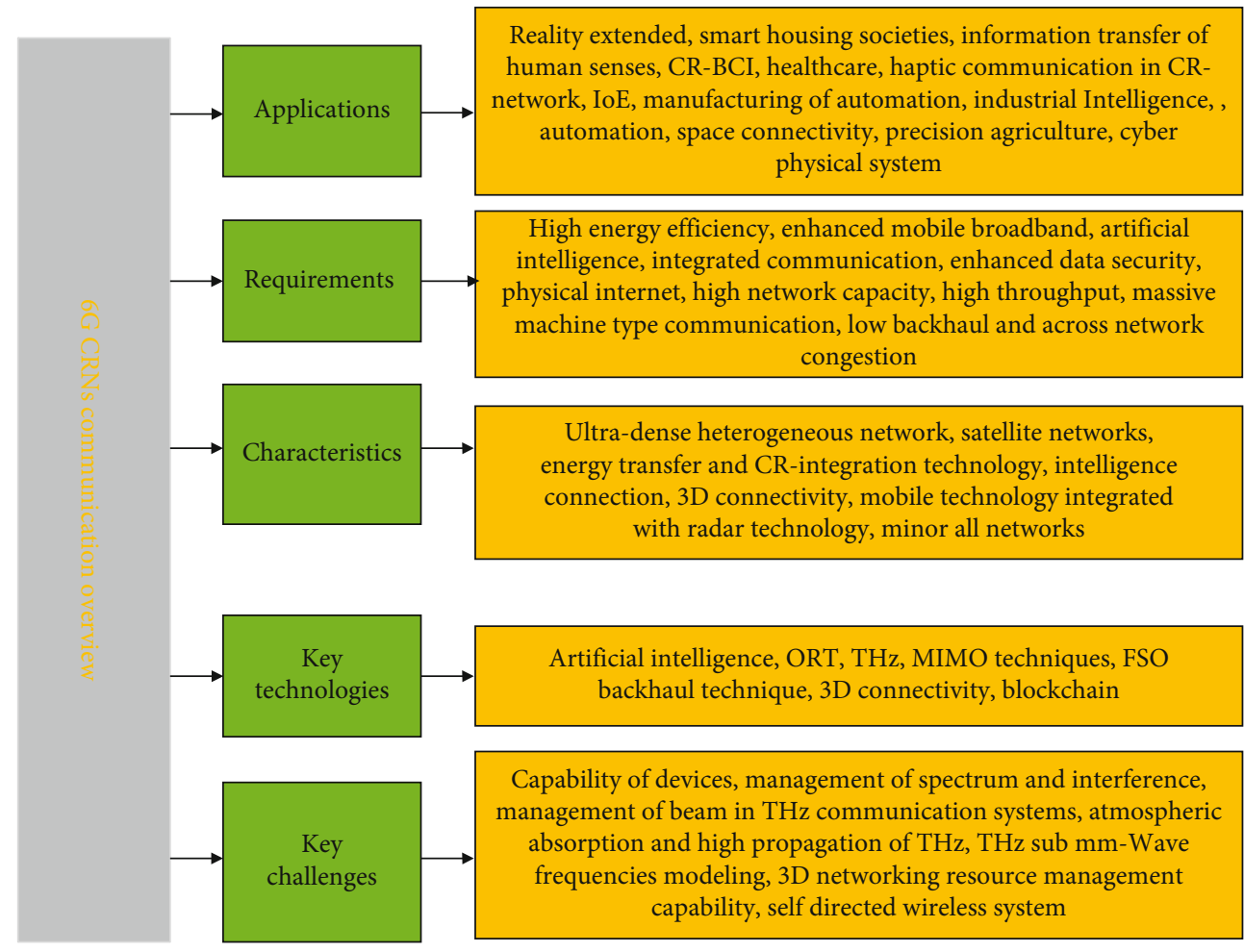

FIgURE 3: Overview of 6G CRN network communication.

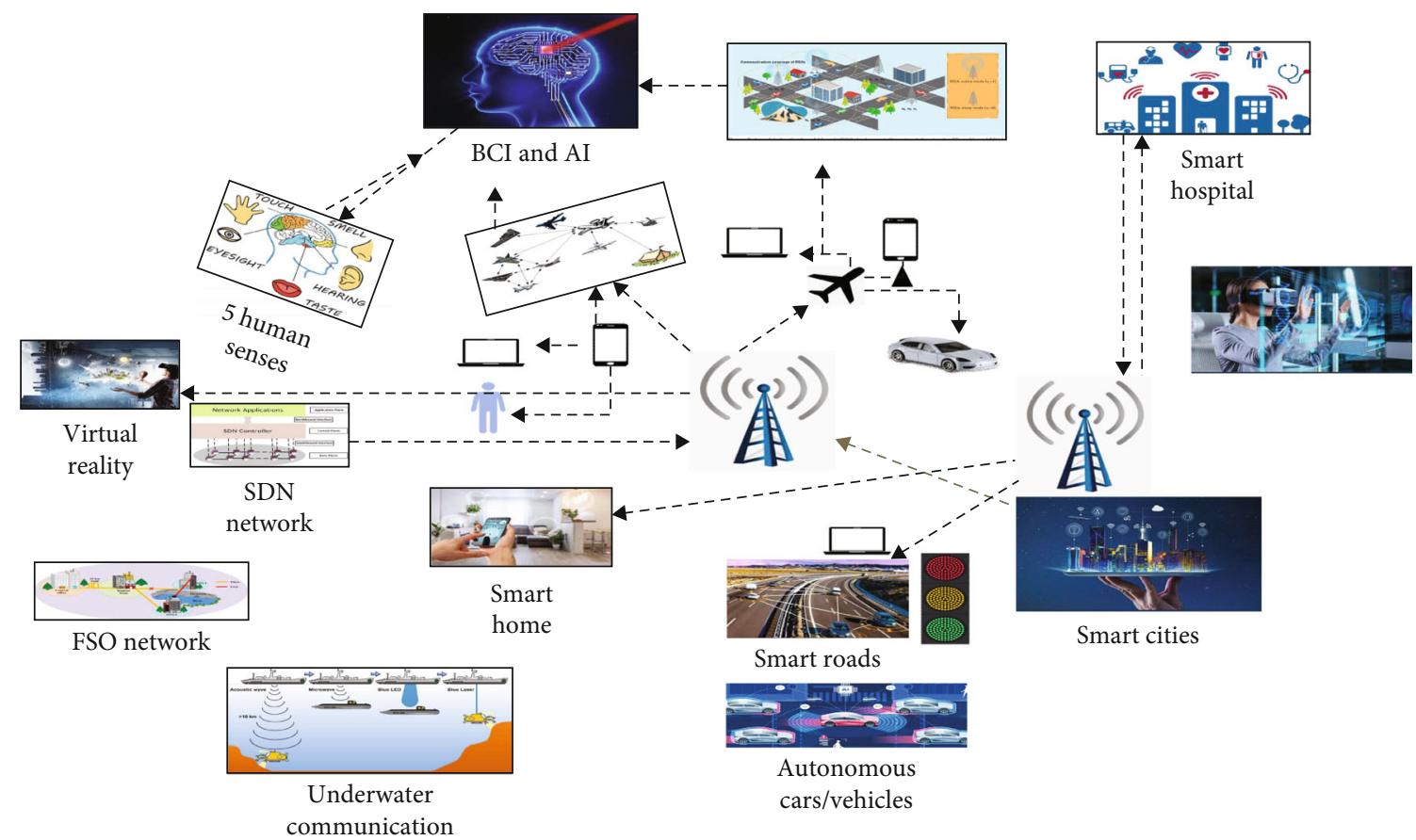

FIGURE 4: Predicted 6G CR network communication.

to-machine (M2M) communication by building intelligent societies. The 6G CR network connection will make our living standards and society super bright by using autonomous vehicles, robotics, mobile devices, etc. Furthermore, the large num- ber of significant and rushed capitals in the globe will organize flying taxis based on 6G CR network connectivity. There will be a reality in intelligent homes because a smart deviceprovided command will control devices in remote access. 
4.3. Information Transfer of 5 Human Senses. To experience the world's surroundings, there are five human senses: sight, touch, hearing, smell, and taste. These five senses received data will be remote transfer in 6G CR network communication. The neurological process by sensory integration is used in this process that detects sensation from the environment and the human body and effectively uses the body in the atmosphere and local environments. Brain-computer interface (BCI) technology will excellently boost this application.

4.4. CR Brain-Computer Interaction (CR-BCI). BCI controls daily used appliances in innovative societies, specifically medical systems and home used appliances. It represents direct communication between external devices and the brain. It acquires brain signals which send to the digital devices, interpret, and examine the signals into other movements. Topographies of $6 \mathrm{G}$ CRN communication will provision actual BCI execution for quality of living standards.

4.5. Healthcare. Medical technology will benefit from 6G CR technology because innovation in VR, XR, MR, AR, mobile edge computing, telepresence, holographic, and artificial intelligence will assist in building intelligent healthcare systems. In the healthcare system, a satisfied remote-control system will be served by $6 \mathrm{G}$ technology. With the use of $6 \mathrm{G}$ technology, there is the possibility of remote surgery. Low latency, high data rate, and feasible 6G CR network will assist fast and promising transport large volume of medical data, which may progress both quality and access to care.

4.6. Haptic Communication in CR-Network. It is the branch of nonverbal communication in which touch sense is being used. Considered 6G CR network communication will care such haptic communication, e.g., the remote user will feel feasibility and happy haptic in actual-time activity. Such system applications and implementation are predicted to be supported by the higher features of 6G CRN communication $[45,46]$.

4.7. Internet of Everything ( $I o E)$. IoE is autonomous coordination and unified integration among many elements (computing elements), people, objects, devices or sensors, and the internet using data and processing. There will be full support of IoE in 6G CR network communication [47]. It is a form of the internet of things (IoT) [22]. Still, a covered-up period that participates following features people, data, physical devices, and processes in a single form. IoT is about objects and communication with each other and physical devices. At the same time, IoE presents an intelligence network to gather all physical objects, data, processes, and people in a single system. IoE in the future will be helpful for innovative health, intelligent societies, and intelligent cars $[21,48]$.

4.8. Manufacturing and Automation. AI-based automation will be available in 6G technology. Automation term suggested to processes automated control systems and devices. The automation system in $6 \mathrm{G}$ will provide secure, scalable, highly reliable, and feasible CR communication using low latency and high data rates.
4.9. Industrial Intelligence Automation. For several years, 4.0 industry is working behind the automation industry based on supply chain optimization notation, additive manufacturing, autonomous equipment, IoT, and data analytics [49]. Still, such notations are treated as storage tower functioning in separation and boundary the real industry automation. In another way, the next feature, industry X.0, seems to feel interactions between different gradations of industrial automation by AI usage. Modern industrial floor with 6G CR communication is expected to need feasible high throughput connectivity across millions of devices with submillisecond delay/response. They are making it suitable for the upcoming $6 \mathrm{G}$ CR communication $[50,51]$.

4.10. Space Connectivity. Deep space connectivity near the earth is still budding with $5 \mathrm{G}$; several use forms range from remote sensing and radio astronomy to backhauling and navigation, which will advance from universal connectivity supported by $6 \mathrm{G}$ technology. Furthermore, such application contains terrestrial cellular offloading, freight tracking, UVA long-range condition, and atmosphere monitoring, to a few names. At the end of this, the internet of space will serve as the main prospects technology for beyond connectivity for 6G CRN technology [52].

4.11. Precision Agriculture. Soil humidity measurements have been a backbone in irrigation verdicts for periods now inside the larger precision agriculture domain. Though, real-time extents and irrigation automation keys still face challenges curtailing strong CR communication coverage deficiency. Working beyond modest automated irrigation explanations, high-performance accuracy agriculture is principally centered around bringing data-driven visions to address the precise needs of farms, customers, soil, and crop. Simultaneously, scalable and appropriate access to such data is a crucial challenge due to breaches in rural connectivity. Consequently, there is a consideration that 6G CR network systems, with their attention on ubiquitous CR, will play a key role in attracting $6 \mathrm{G}$ technology in the agricultural invention.

4.12. Cyber-Physical Systems (CPS). UAVs and autonomous cars are about the most auspicious CPS in the present day. Such autonomous system process is characterized by the conversation of significant amounts of data amid the integral nodes, i.e., UAVs and cars, concerning high-resolution realtime planning of the topography, optimization, route, and traffic, and security information. Though the resulting bulky volumes of data must be transported inside firm deadlines in an error-free means, this is also authoritative to note that such nodes characteristically work at speeds in surplus of $100 \mathrm{~km} / \mathrm{h}$. Furthermore, to provide very high reliability, submillisecond latency and the connectivity solution that allows autonomous CPS necessity also bid healthy processes at high speeds, which seems impossible with present 5G systems and technology [53].

Some other applications are as follows

(i) 3GPP technologies

(ii) Inclusive sensing 


\section{(iii) Communication-based on beam forming}

Figure 5(a) is representing CR network environment, (b) is representing $5 \mathrm{G}$ CR Network requirements, (c) is representing features or advantages of 5G CR network, and (d) is representing $6 \mathrm{G}$ intelligent network applications. It is showing connectivity of various applications, usage, and control in one hand. Everything is connected with internet. There seems access of internet and connectivity in 6G intelligent applications.

\section{Security Issues of CR Network in 6G Technology}

In $\mathrm{CR}$ networks, privacy protection is a basic need in $6 \mathrm{G}$ technology. In CRNs, data can be lost or stolen and can be physically attacked. CRNs are more sensitive in security threats than traditional wireless networks, because in CRNs, there seems no proper cooperation in between PU and SU communication. Several security issues caused by CR, because CRN environment is sensing information based, can vary node behavior. As learning is one of the basic characters of CR, because of this having wrong supposed environment, may cause to give error in new decision. In resultant, malicious attack will take advantage for longterm effect on behavior.

Data gathered by CR can be destroyed, sniffed, or change by illegal entities. Furthermore, attackers may disturb transmission of PUs or protect channel usage by SUs by spectrum sensing data falsification. There must be acceptable level of security robustness against attacks and threats in CRNs. There are further security issues in 6G CRNs, some of issues are following, modification of data, private data access, and improper interruption to PUs, barring use of common control channel by artificially yielding blockage issue, barring use of idle channel for unlicensed users, and false data injection. We studied about 5G and 6G CR networks. In 5G, some technologies are helpful in 6G areas. 5G technologies give low latency, high reliability, efficient transmission, and secure services to $6 \mathrm{G} \mathrm{CR}$ networks. In $6 \mathrm{G}$, an extremely large number of small amounts of data connections may cause a high risk of people's security and privacy with comprehensive care attracted by commerce, business, and governmental entities. The more prominent risk they may execute on securing client protection and causing regulatory problems. So here, we study some fundamental security issues and possible attacks in 6G CRN technology.

5.1. Artificial Intelligence. In 5G CR networks, artificial intelligence works in separate areas where a huge quantity of robust and training data and hidden nodes also exist. AI will play an important role in $6 \mathrm{G}$ wireless networks. There is a greater impact of machine learning and AI technologies on privacy in two ways. First, the right applications of machine learning may improve $6 \mathrm{G}$ privacy, while second, on machine learning attacks, privacy defilements may occur. When AI transfers to the network edge, more sophisticated applications will work on devices, increasing attack threats. Although, integrating privacy-protecting mechanisms in resource-constrained devices will be stimulating. While in 6G, AI can be divided among several layers, such as computing layers (network function virtualization, software design network, and edge computing) and physical layer (data link and network architecture devices). There may be security issues of malicious behavior, control access, communication, and authentication, etc.

5.2. Molecular Communication. In molecular communication, communication is done by using molecules in an aqueous environment or chemical signals-enhanced technology for $6 \mathrm{G}$ in several healthcare applications such as wearable body sensors and telemedicine. The use of CR wearable body wireless sensors [54] may mitigate such issues because of global operability, bandwidth, and jamming and enhance consistency. Highly sensitive information is tackled by molecular communication with many privacy concerns related to the encryption process, authentication, and communication. However, providing safe molecular communication is important. In [55], biochemical cryptography has been introduced in which a biological structure and macromolecule composition have been used as a medium to maintain information integrity. In diffusion-based channels, physical layer security and primary benefits are studied in $[56,57]$, where privacy ability has been discussed to gain insights on protecting symbols a diffusion-based channel can afford. For molecular communication, security needs more research and practical work. In 6G molecular communication, there are several attack methods such as de synchronization, flooding, and jamming attacks. CR implementation requirements in wireless medical networks have been discussed in $[7,58,59]$.

5.3. Blockchain Privacy. Blockchain privacy and security problems are associated with the communication process, access control, and verification. Recently, in the telecommunication industry, blockchain gained much more attention. Benefits of blockchain like nonreputation, disintermediation, proof of provenance, immutability, pseudonymity, and integrity are particularly important to enable various facilities in 6G technology with privacy and security. The use of AI and some analytic technologies may cause attacks such as evasion attacks in the testing process and poisoning attacks in the training process. Blockchain can guard against protecting the reliability of AI data via distributed trust and immutable records between various shareholders, with assurance in the AI system in multitenant. In $6 \mathrm{G}$ networks, this trust gives the required confidence for clients to adopt AI-based security management systems, and it may be insufficient in preventing their failure and breach in AI systems. Blockchain-based projects can be used in trust-level agreements in the liability of each party. In [60], authors studied blockchain radio access network architecture to effectively manage and secure network verification and access between trustless objects. In [61], authors studied to enhance the security of CRNs by a blockchain method to contact vacant license spectrum. In addition, to support blockchain role to adjust with $6 \mathrm{G}$ necessities, several current $5 \mathrm{G}$ models need active involvement. Blockchain plays an important role in 


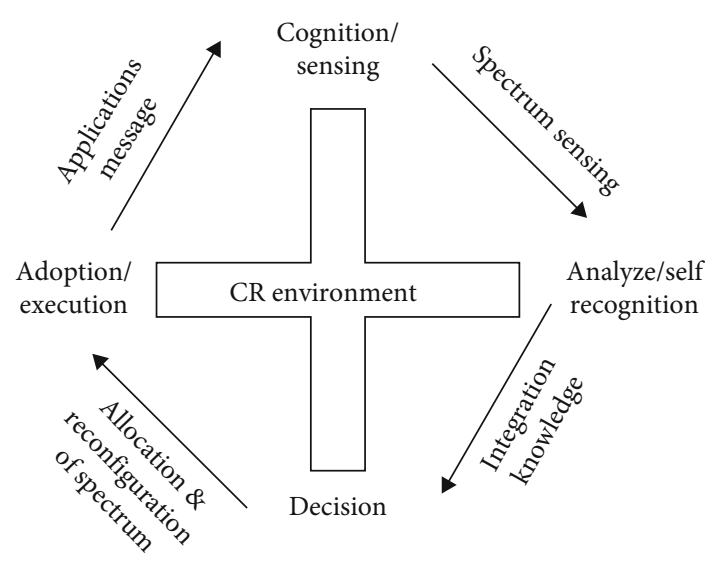

(a)

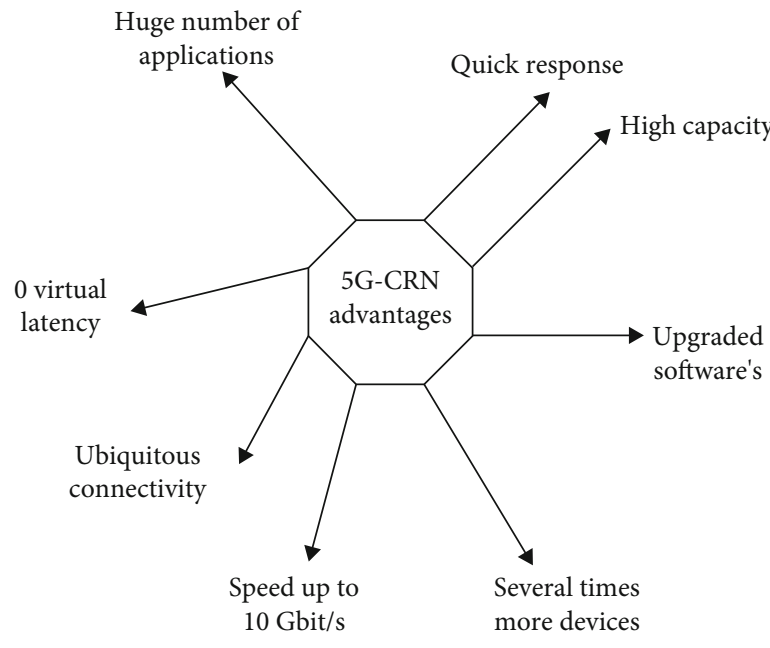

(c)

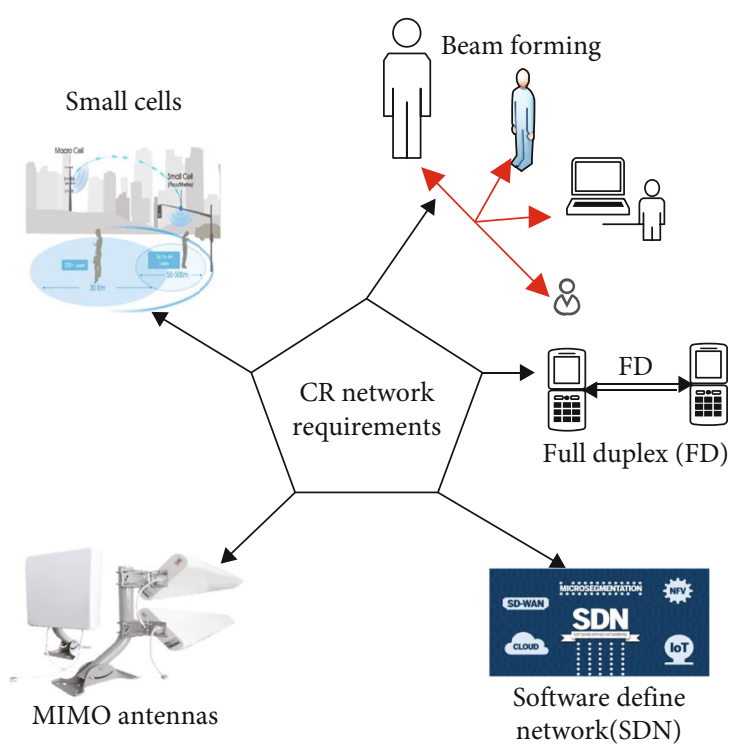

(b)

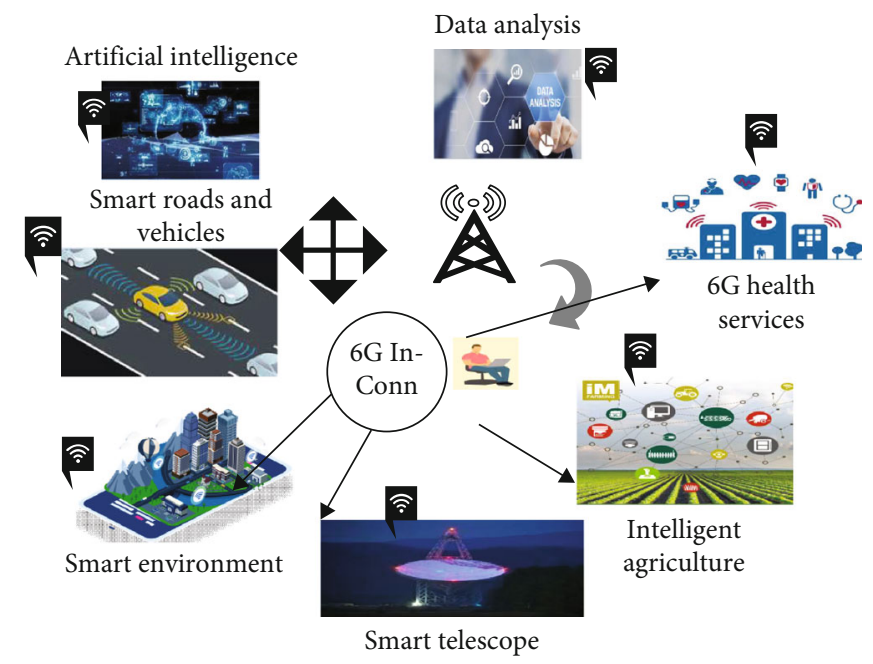

(d)

FIGURE 5: CR network environment, requirements, 5G features, and applications. (a) CR network environment. (b) CR network requirements in 5G. (c) Advantages of 5G CRNs. (d) Applications of 6G intelligent network.

privacy protection in $6 \mathrm{G}$ networks. In blockchain, having mutual communication channel may permits users to be recognized by pseudoname instead of personal identities.

5.4. Security Concerns in $\mathrm{THz}$. $\mathrm{THz}$ communication such as $\mathrm{GHz}$ to $10 \mathrm{THz}$ is intended as an important technology for $6 \mathrm{G}$. In these frequencies, improved directionality of transmitted signal is present, limiting illegal clients for signals interrupting and providing high security at the physical layer. Interception of signals by eavesdroppers has been proved in [62]. In 6G CRNs, $\mathrm{THz}$ also has security issues focus on malicious and authentication behavior. In [63, 64], authors studied $\mathrm{THz}$ frequency electromagnetic signature and can be used for verification procedure of physical layer. [27, 28] authors studied that an eavesdropper could still interrupt a signal when conveyed via a narrow beam. Certainly, $\mathrm{THz}$ communication is disposed to access data transmission exposure, control attacks, and malicious behavior. For the security of $\mathrm{THz}$ communication, new physical layer systems are needed.

5.5. Physical Layer Attack. On physical layer, jamming attack is known as one kind of attack. In such kind of attack opponent sends radio signals on wireless channel to interrupt CRN working position. Such opponent may have sophisticated and powerful software and hardware. If entire network is blocked by opponent, it found DoS attack on physical layer attack. On physical layer attack another kind of attack is tampering attack. Here, attackers can damage CRNs, replacing hardware parts or whole nodes.

5.6. Routing Layer Attack. In this type of attack, attackers misguide the packet and change information on routing packet forwarding sensors on networks. Similar may happen 
to data packets as well. Sybil attack, wormhole attack, and sinkhole attacks are possible in CRNs.

5.7. MAC Layer Attack. In such attack, attackers violate defined rules and create disturbance in normal operation. Such as transferring unwanted packets frequently to exhaust battery power and disturb normal operation with the use of selfishly channel and present no cooperation on priority of MAC cooperative layer. This may also lead to DoS attack.

\section{Requirements for $6 \mathrm{G}$ CR- Network Technology}

There are many problems in the 5G trade-off, such as energy efficiency, throughput, delay, reliability, hardware complexity, and deployment cost. This is to be considered that $5 \mathrm{G}$ is not meeting the market and users' requirements of 2030 . There is another option of 6G, which may fill such a gap of user requirements. Based on previous work and upcoming requirements, the key objectives for $6 \mathrm{G}$ are millions of connected devices, ultrahigh reliable connectivity, very high perdevice data rate, extremely low latency, global connectivity, and reducing energy use with battery-free IoT devices [65].

6.1. Requirements. Future CR network communication will have the following basic requirements.

(i) High energy efficiency

(ii) Enhanced mobile broadband

(iii) Artificial intelligence integrated communication

(iv) Enhanced data security

(v) Physical internet

(vi) High network capacity

(vii) High throughput

(viii) Massive machine-type communication

(ix) Low backhaul and access network congestion

It is to be considered that there will be 1000-time higher simulation in 6G CR networks than 5G. URLLC, a basic feature of $5 \mathrm{G}$ will also play an important role in $6 \mathrm{G}$ communication by providing less than $1 \mathrm{~ms}$ end-to-end E2E delay. In $6 \mathrm{G}$, volume spectral efficiency will be better than $5 \mathrm{G}$. There will be the capacity of extended battery life in $6 \mathrm{G}$ technology. There will be no need to charge mobile devices in 6G technology separately.

\section{Characteristics of New Network}

7.1. Ultradense Heterogeneous Network [66]. Such networks will play an important role in 6G CR network communication. These networks will reduce the cost and improve the quality of services.

7.2. Satellite Networks. For worldwide devices, connectivity, $6 \mathrm{G}$ is predictable to integrate with satellite systems. Integra- tion of satellite, air-bone networks, and terrestrial into one CR network system will be key in $6 \mathrm{G}$.

7.3. Energy Transfer and CR-Information's Integration. In 6G technology, the CR network will send power to battery devices like sensors and devices. Since energy transfer and CR network will be integrated.

7.4. Intelligence Connection. Contrary to the traditional generation of CR networks systems, upcoming technology like $6 \mathrm{G}$ and beyond will be transformative and will update $\mathrm{CR}$ improvements from linked intelligence to linked things. There will be the introduction of AI in all communication steps. There will be the new paradigm of AI in the CR communication systems.

7.5. 3D Connectivity. In wireless communication, UAV-based devices provide suitable solution to enhance connectivity whenever traffic data is nonstationary and heterogeneous, and it is to be considered maximum varying with time or space. To get access to various UAV-assisted applications, UAV requires connection or communication with present network either Wi-Fi or cellular, in this case, UAV response as UE of network. UAVs can response as UE applications, e.g., UAV-assisted transportation network, UAV-based drones, and real-time surveillance UAV devices used as relays to provide connectivity between aerial base station and aerial UEs. Retrieving the network and central network purposes low earth orbit satellite and drones will enable super $3 \mathrm{D}$ connectivity in $6 \mathrm{G}$ technology.

7.6. Mobile Technologies Integrated with Radar Technology. To gain maximum precision localization with CR communication and key concept of 6G CR networks, communication $6 \mathrm{G}$ will be integrated with radar [67].

7.7. Minor Cell Networks. Basically term minor or small cell is compact to low power base stations $(10 \mathrm{~W}-20 \mathrm{~W})$, such as femto cells, micro cells, and Pico cells. On another hand, ultradense network (UDN) is extended form of heterogeneous network in which huge number of small cells is deployed to reduce traffic from highly dense macro cells and network performance can be enhanced. UDN technology is one of the important technology which helps in network performance like latency, capacity, and data rate. In a UD network, overdeployment of small cells increased resource management challenges with respect to energy efficiency, spectrum resource efficiency, and control overhead, e.g., comparing to macrocells base station small cell microstation uses less energy on communication and operation. Minor cell network notation has been initiated to improve signal quality as a sign of energy efficiency, throughput, and spectral efficiency improvement on the cellular network. As a result, minor cell networks are necessary for $5 \mathrm{G}$ and $6 \mathrm{G} \mathrm{CR}$ network communications. Figure 6 is showing mmWave massive MIMO-based ultradense network for $6 \mathrm{G}$ communication. 


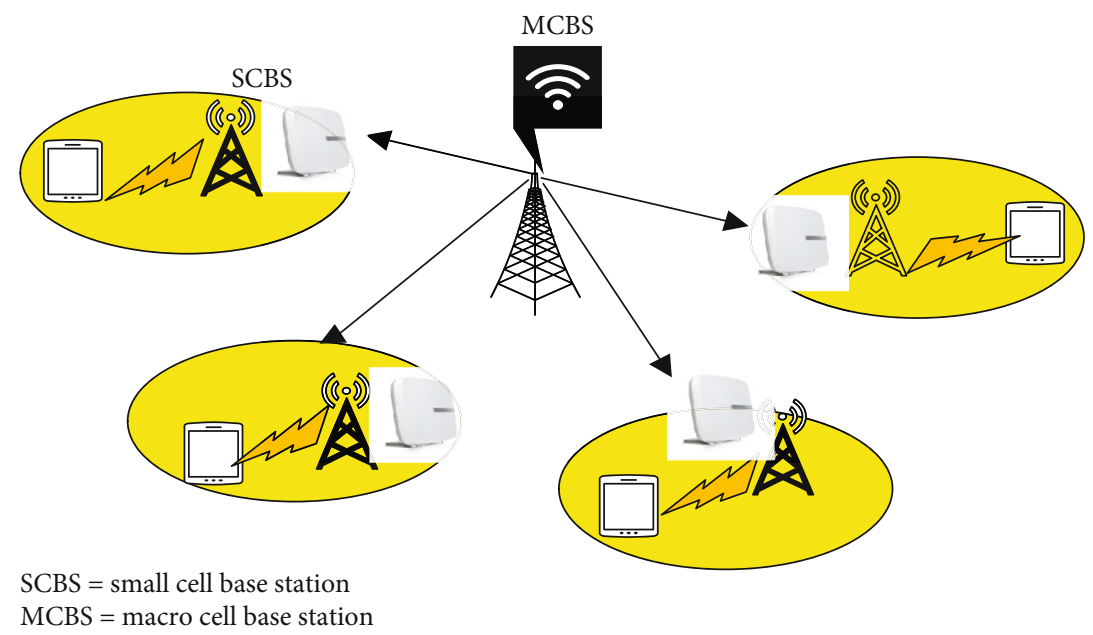

Figure 6: Massive MIMO-based ultradense network for 6G.

\section{Key Technologies for 6G CR-Network}

6G CR-Network will be driven by several technologies; some of the key technologies are studied below.

8.1. Artificial Intelligence. An essential and introduced technology in 6G CR-communication is $\mathrm{AI}[68,69]$. In $4 \mathrm{G} \mathrm{CR}$ network systems, AI was not deployed. At the same time, $5 \mathrm{G}$ is partially supporting AI technologies. Although, $6 \mathrm{G}$ is predicted to support AI for automation services [33] fully. Machine learning improvements will provide a more intelligent CR network in 6G for real-time communication. In CR-communication, AI introduction will simplify and make real-time data improvement. With the use of several analytics, a complex target way can be determined by AI.

(i) AI will be helpful in reduction of processing delay and increasing efficiency of CR-communication steps

(ii) AI will be helpful in time-consuming tasks, like network selection and handover

(iii) There will be the key role of $\mathrm{AI}$ in $6 \mathrm{G}$ in $\mathrm{M} 2 \mathrm{M}$, $\mathrm{M} 2 \mathrm{H}$, and $\mathrm{H} 2 \mathrm{M}$ communication

(iv) $\mathrm{AI}$ will rapid $\mathrm{CR}$-communication in $\mathrm{BCI}$

CR-network communication systems based on AI will be maintained by intelligent structure, intelligent devices, metamaterials, self-sustaining CR networks systems, intelligent cognitive radio, machine learning, wireless networks, and intelligent networks $[70,71]$.

8.2. Optical Radio Technology (ORT). This technology is envisioned for 6G CR communication technology [72], in addition to $6 \mathrm{G}$ radio frequency (RF) based communication for all predicted devices to access network, and such networks also access network to fronthaul/backhaul network connection. This technology is also functioning since $4 \mathrm{G}$ CR-communication systems. Although, it will be widely used for 6G CR-communication demands. ORT is like visi- ble light communication, light fidelity, FSO communication, and optical camera communication. Researchers are focusing on overcoming challenges and enhancing performance in present technologies. Optical radio technology communication provides low latency, secure communication, and high data rates. LiDAR is also an optical band which is the key technology for $3 \mathrm{D}$ resolution in $6 \mathrm{G} \mathrm{CR}$ network communication.

8.3. Terahertz Communication. Spectral efficiency can be enhanced with the bandwidth increase; it is possible to do it by sub- $\mathrm{THz}$ communication with max bandwidth and apply advanced MIMO networks. The radio frequency band has already been bushed, and this time, it is not sufficient to meet $6 \mathrm{G}$ requirements. There will be the key role of $\mathrm{THz}$ bands in the $6 \mathrm{G} \mathrm{CR}$-communication network [73, 74]. Such band is intended to be the next limit of maximum data rate CR communication system. $\mathrm{THz}$ waves are known as submillimeter radiation, generally state to $0.1 \mathrm{THz}$ and $10 \mathrm{THz}$ frequency band with 0.03 to $3 \mathrm{~mm}$ corresponding wavelength range $[64,69]$. According to the ITU-R recommendation, the $275 \mathrm{GHz}-3 \mathrm{THz}$ band range is considered the central part of the $\mathrm{THz}$ band for CR communication [69]. $6 \mathrm{G}$ cellular communication will be enhanced by accumulation to $\mathrm{THz}$ bands $275 \mathrm{THz}-3 \mathrm{THz}$ still have not been assigned for any purpose globally, while such band can approach high data rate [73]. By adding this $\mathrm{THz}$ band with the present mmWave band, the total band capacity increased about 11.11 times. So, the $300 \mathrm{GHz}-3 \mathrm{THz}$ band represents resemblances with the radio frequency band. $\mathrm{THz}$ improves the potential and high-frequency challenges in CR communication systems [75].

There are few basic properties of $\mathrm{THz}$ communication, such as

(i) High frequency raised high path loss

(ii) For support of high data rate, widely bandwidth available

Highly directional generated narrow bandwidth antennas decrease the interferences. $\mathrm{THz}$ small wavelength 
permits a more significant number of antennas to join into devices and base stations functioning in such band. It permits the use of advanced adaptive array technologies which overcome the limitation.

8.4. MIMO Technique. One primary technique is the MIMO technique application [76-78]. With the improvement of MIMO techniques, spectral efficiency directly improves [79]. Therefore, massive MIMO technology will be helpful in $6 \mathrm{G}$ communication networks.

8.5. FSO Backhaul Technique. It is not possible that the backhaul network has optical fiber (OF) connectivity because of inaccessible terrestrial locations and complications. The FSO backhaul network is very encouraging for 5G CR network communication systems [80, 81]. The transmitter and receiver features of the FSO system are similar to those of OF networks. Thus, data transfer in the FSO system is analogous to the OF system. Though, laterally with the OF networks, FSO is an admirable technology to provide backhaul connectivity in $6 \mathrm{G}$ communication networks. With FSO, there is the possibility to have pervasive range communications even at a $10,000 \mathrm{~km}$ distance. FSO provisions highcapacity backhaul connection for distant and nonremote areas, like outer space, sea, isolated islands, and underwater; FSO also provisions cellular base station connectivity.

8.6. High Visibility 3D Connectivity. 6G CR communication will integrate airborne and ground networks for user's communication provision. The $3 \mathrm{D}$ base stations will be provided over UAVs and small orbit satellites. The accumulation of original proportions concerning elevation and related degrees of liberty makes $3 \mathrm{D}$ connectivity significantly diverse.

8.7. Blockchain. It will be an imperative technology to accomplish big data in upcoming communication system networks. Blockchains are objective one system of the distributed ledger technology. At the same time, a distributed ledger is defined as that it is a database that is distributed across several nodes. Each node duplicates and protects a similar copy of the ledger. Peer-to-peer networks accomplish the blockchain. It can occur deprived of being achieved by a centralized authority. Data on a blockchain is collected, organized, and structured in chunks. The chunks are linked to one another and tenable using cryptanalysis. The blockchain is fundamentally a faultless accompaniment to the vast IoT with enhanced security, interoperability, reliability, privacy, and scalability. So, such technology will afford numerous services, like traceability of massive data, interoperability across devices, autonomic contacts of diverse IoT systems, and consistency for the enormous connectivity of $6 \mathrm{G} \mathrm{CR}$ communication systems.

Other key enabling technologies include quantum communication, unmanned aerial vehicles, communication, sensing integration, dynamic network slicing, accessbackhaul network integration, holographic beam farming, energy transfer, wireless information integration, and cellfree communication big data.

\section{Key Challenges and Future Discussion on 6G CR Network Communication}

Nowadays, Information and Communication industry is facing worldwide challenge, such as new services development with enhanced QoS and on the spot less environmental effect. There is gap between demand and supply of wireless [82]. There seems fear that imminent spectrum crises where increase requirement from smart mobile devices will soon overcome wireless capacity may occur. Challenge is deficiency of new spectrum present in wireless data carrier. Data traffic of smartphones is increasing speedy that if nothing is done they can use present spectrum. Such thing may not affect only phones but also other used wireless equipment.

To study efficiency of spectrum usage problem, CR idea was given. This CR technology has potential of being a troublesome force within spectrum management [83, 84]. Famous example is opportunistic spectrum or opportunistic radio those rules are spatial, temporal, and geographic reuse of legal spectrum. Several use of spectrum appearing that spectrum is in use, in form that complicated responsibility of spectrum usage at unplanned geographical location and at fixed frequency is not much high. It shows that there are several holes in radio spectrum which could be oppressed. Opportunistic radio system must have ability to feat such spectrum holes by detection and use in opportunistic way. Because of such propagation in television bands with floor penetration ability and strong wall, flexible bandwidth and long range may use to permit new services and enhance bounded capacity of present systems $[85,86]$.

On the other hand, CR is not bounded to opportunistic spectrum access and contains heterogeneous network [87]. Present spectrum locations are not permitted for frequency bands allocation to various radio access technologies [88]. Such technology may open several new applications and available opportunities, like city and campus coverage, rural connectivity, massive wireless hotspots, and content distribution networks [89].

There are several challenges facing in CRNs in both hardware and network architecture. For CRN network, it needs several technologies, like communication pattern learning intelligence, radio environment awareness, and robustness of spectrum use. Here, CRNs must be effective in network capacity as well as spectrum utilization. Coexistence and interoperability between several communication standard challenges are present. Huge quantity of data produced by huge number of real-time device should be adjusted by CRNs, such as low latency. For enhancement of network performance, CRN architecture has been executed. Numerous technical complications want an effective solution in the $6 \mathrm{G}$ installation of CR networks. Possible predictable challenges in 6G are studied below [86].

(i) Switching protocols b/w CR function protocol $[90,91]$ (battery power devices, e.g., LPWAN)

(ii) Access strategy for IoT wireless sensors network. (Huge number of users are responsible for dispute b/w secondary users (SUs), so spectrum sharing 
strategy is essential here for successful management of several kinds of sensors access)

(iii) Interference sensing channel coding [91-93] (challenge of selecting optimal channel coding technique, which matches SUs QoS and not preventing primary users (PUs) simultaneously)

(iv) Using smart reflecting surface (noncooperation situation between PU and SU, complicated to apply channel estimation. Cascade and blind channel estimation could be helping SRS applications for the help to spectrum sensing (SS)) [94]

(v) Exchange protocol for wireless sensor networks [95] (data sending nodes should be up-to-date by a central entity about already present channel)

(vi) For CR, sensing spatial dimension [96, 97] (in noncooperation manner with $\mathrm{PU}$, estimation beam of PU become a challenge, even to know PU, SU beam adjustment is also challenging because of secondary user transmitter inevitable interference to $\mathrm{SU}$ receiver)

(vii) SS binary decision complication indirection of smart spectrum sensing [98]

(viii) In CR communication technology, there is a challenge of future public safety $[99,100]$ such as license plate detection, web access, and video streaming

(ix) The primary key challenge in CRN is the demonstration of CRN technology, assess, and failure to conclusively test at real-world installation scenario and scale. Physical installation needs a high resolution, speedy signal processor, and high sampling rate for CR applications in cooperative sensing $[101,102]$

(x) Challenge when SU does not see PU [12]

(xi) During SUs transmission, PUs can claim their licensed band, and in this process, there may be the challenge of sensing duration as studied in $[12,103]$

(xii) Economics and spectrum policy challenges [104] such as the challenge in conveying energetic spectrum standards are that of essentially progressing spectrum utilization effectiveness without losing the benefits of inactive spectrum assignment [27]. The challenge in installing wireless equipment and networks that can unscrupulously work in various frequency bands and spectrum policy domains is challenging $[105,106]$

Future discussion and some more key challenges are discussed below.

9.1. The Capability of Devices (Smartphone). There must be several advances and improved features in upcoming technology. There should be the ability of intelligent devices to adjust to the upcoming technology and its features. It does not seem very easy to provision throughput $1 \mathrm{~Tb}, \mathrm{XR}$, inte- grating sensing, and AI with CR network structures using specific devices. There are few chances of currentgeneration $5 \mathrm{G}$ that could not provision some upcoming generation $6 \mathrm{G}$ features. Also, ability enhancement in advance (6G) devices might raise the price. Billions of devices are associated with fifth-generation (5G) technology this time; so, it needs to be assured that 5G-associated devices are suitable for $6 \mathrm{G}$ or not $[72,106]$.

9.2. Management of Spectrum and Interference. Because of the privacy of the interfering problems and spectrum resources, there is significant to professionally accomplish $6 \mathrm{G}$ ranges with the sharing of spectrum tactics and advanced spectrum management techniques [27]. For attaining supreme resource operation with QoS expansion, wellorganized spectrum management is significant in this case. There needs concentration of researchers towards 6G, like spectrum sharing and spectrum management procedure in various networks which harmonize communication at a similar frequency. There is a need to examine the procedure for canceling interference using standard interfering cancellation methods, like successive interference cancellation and parallel interference cancellation.

9.3. Management of Beam in $\mathrm{THz}$ Communication Systems. In a massive MIMO system, beamforming is capable technology for the provision of speedy data rate communication. But in THz, beam management is not easy, and it is complicated or challenging due to sub mmWave propagation. Though, selecting optimal beam efficiently is essential in speedy traffic management.

9.4. Atmospheric Absorption and High Propagation of Terahertz $(\mathrm{THz})$. High $\mathrm{THz}$ frequencies provide high data rates. Though, there is essential for $\mathrm{THz}$ to defeat key problem for transferring data on comparatively extensive distances for atmospheric absorption characteristics also high propagation loss. For the $\mathrm{THz}$ communication network, we need a novel design for transceiver architecture. The transceiver must work at high frequencies and want to confirm the complete use of extensively existing bandwidths. Trivial effective area and gain of different $\mathrm{THz}$ band antennas are the alternative issues of $\mathrm{THz}$ communication. Safety and health anxieties associated with $\mathrm{THz}$ band communications are also essential to be discussed.

9.5. THz Sub-mmWave Frequency Modeling. Sub-mmWave $(\mathrm{THz})$ and mmWave broadcast features focus on atmospheric situations; consequently, diffusing and absorptive properties have been observed. The extreme situation usually is variable and thus moderately impulsive. So, such band channel modeling is comparatively complicated also; there is no perfect channel model in this band.

9.6. 3D Networking Resource Management Complexity. There is speedy progress towards $3 \mathrm{D}$ networking. Though, there was an increase in novel addition. Furthermore, numerous opponents may interrupt truthful information that may meaningfully damage the general system performance. So, novel techniques for optimizing routing protocol 
and resource management, multiple access, and mobility support are essential. Preparation desires a novel CRN project.

9.7. Self-Directed Wireless Systems. There will be a 6G system for self-directed or automatic technology like UAVs, selfdirected car, and AI-based industry. We want to have junction of numerous assorted subsystems for autonomous wireless networks, like interoperable processes, machines of systems, autonomous computing, machine learning, systems, autonomous cloud, and heterogeneous CR networks. Therefore, general system progress becomes challenging and complex. e.g., emerging an entirely autonomous system for the driverless car will be more challenging because researchers in $6 \mathrm{G}$ require to project fully automated selfdriving cars that achieve better than human-controlled cars [107].

In 6G CRN communication technology, it may answer the following questions

(i) How much bandwidth assigned to each SUs, and who is willing to use the existing spectrum hole of different networks?

(ii) What is the power assigned to SUs?

(iii) During the switching process of SU from network to network, is the SU compatible for dynamic acceptance of network limitation variations?

(iv) For SUs support in the CRN environment, which mobility model could be best?

\section{Conclusion}

With time, technology is progressing day by day and generation to generation. 5G CR network communication is functioning in several big cities of developed countries and spreading globally. Although, 5G is not fulfilling the requirements of industries and users for the CR network in 2030. So, 6G may cover several requirements of 2030 . It is to be expected that $6 \mathrm{G}$ wireless network will support client's applications and enhance communication latency with high throughput and low latency. This article explained review on upcoming generation of CR network communication, mentioned mandatory cases, and detailed prospective on present and future technology improvement efforts. We studied the application and requirements for 6G CR network communication. In the end, we also highlighted the possible key challenges in 6G CR network communication and significant empowering technologies that are energetic to the achievement of $6 \mathrm{G}$ and beyond. Besides illuminating the visualization and goal of $6 \mathrm{G}$ CR network communications, we have quantified the numerous technologies that could prove helpful for $6 \mathrm{G}$ CR network communication.

There are also some limitations in 6G technology, such as it has been studied in this paper that $6 \mathrm{G}$ uses multiconnectivity and cell-less technology. For this, network requires perfect architecture. Design of new cell-less architecture is challenge. Moreover, there are challenges or limitations is
$6 \mathrm{G}$ to use $\mathrm{THz}$ frequencies, visible light frequencies, and energy harvesting requirement.

We also hearten researchers, readers, and book lovers to play their role in realizing upcoming CR network communication technology.

\section{Data Availability}

Data can be provided upon request.

\section{Conflicts of Interest}

We hereby confirm that there is no conflict of interest between authors to declare.

\section{Acknowledgments}

This work was supported by the National Key R\&D Program of China (the major project no. 2020YFB1807900) of China.

\section{References}

[1] D. M. Venkatesan, D. A. V. Kulkarni, and D. R. Menon, "Role of cognitive radio in 5G," Helix, vol. 9, no. 2, pp. 4850-4854, 2019.

[2] M. J. Kaur, M. Uddin, H. K. Verma, and B. R. Ambedkar, "Role of cognitive radio on $4 \mathrm{G}$ communications a review," Journal of Emerging Trends in Computing and Information Sciences, vol. 3, no. 2, pp. 272-276, 2012.

[3] H. Yu and Y. B. Zikria, "Cognitive radio networks for internet of things and wireless sensor networks," Sensors, vol. 20, no. 18, p. 5288, 2020.

[4] J. Adu Ansere, G. Han, H. Wang, C. Choi, and C. Wu, "A reliable energy efficient dynamic spectrum sensing for cognitive radio IoT networks," IEEE Internet of Things Journal, vol. 6, no. 4, pp. 6748-6759, 2019.

[5] Z. Ahmed, M. M. Khan, M. A. Saeed, and W. Zhang, "Consensus control of multi-agent systems with input and communication delay: a frequency domain perspective," ISA Transactions, vol. 101, pp. 69-77, 2020.

[6] A. Srivastava, M. S. Gupta, and G. Kaur, "Energy efficient transmission trends towards future green cognitive radio networks (5G): progress, taxonomy and open challenges," Journal of Network and Computer Applications, vol. 168, article 102760, 2020.

[7] J. Wang, M. Ghosh, and K. Challapali, "Emerging cognitive radio applications: a survey," IEEE Communications Magazine, vol. 49, no. 3, pp. 74-81, 2011.

[8] A. Srivastava and G. Kaur, "Resource management for traffic imbalance problem in green cognitive radio networks," Physical Communication, vol. 48, article 101437, 2021.

[9] S. Bojja Venkatakrishnan, E. A. Alwan, and J. L. Volakis, "Wideband RF self-interference cancellation circuit for phased array simultaneous transmit and receive systems," IEEE Access, vol. 6, no. c, pp. 3425-3432, 2018.

[10] V. Syrjala, K. Yamamoto, and M. Valkama, "Analysis and design specifications for full-duplex radio transceivers under RF oscillator phase noise with arbitrary spectral shape," IEEE Transactions on Vehicular Technology, vol. 65, no. 8, pp. 6782-6788, 2016. 
[11] A. S. Musleh, G. Chen, and Z. Y. Dong, "A survey on the detection algorithms for false data injection attacks in smart grids," IEEE Transactions on Smart Grid, vol. 11, no. 3, pp. 2218-2234, 2020.

[12] M. M. Aslam, L. Du, Z. Ahmed, M. N. Irshad, and H. Azeem, "A deep learning-based power control and consensus performance of spectrum sharing in the CR network," vol. 2021, Article ID 7125482, pp. 1-16, 2021.

[13] X. Li, J. Fang, W. Cheng, H. Duan, Z. Chen, and H. Li, "Intelligent power control for spectrum sharing in cognitive radios: a deep reinforcement learning approach," IEEE Access, vol. 6, pp. 25463-25473, 2018.

[14] Y. Lu, P. Zhu, D. Wang, and M. Fattouche, "Machine learning techniques with probability vector for cooperative spectrum sensing in cognitive radio networks," in 2016 IEEE wireless communications and networking conference, Doha, Qatar, 2016.

[15] X. Qiao, Y. Huang, S. Dustdar, J. Chen, and S. Dustdar, "6G vision: an AI-driven decentralized network and service architecture," IEEE Internet Computing, vol. 24, no. 4, pp. 33-40, 2020.

[16] G. Fettweis, "The tactile internet: applications and challenges," IEEE Vehicular Technology Magazine, vol. 9, no. 1, pp. 64-70, 2014.

[17] M. M. Aslam, L. Du, Z. Ahmed, H. Azeem, and M. Ikram, "Consensus performance of traffic management system for cognitive radio network: an agent control approach," in Cyberspace Data and Intelligence, and Cyber-Living, Syndrome, and Health, Springer Singapore, 2019.

[18] S. Filin, H. Harada, H. Murakami, and K. Ishizu, "International standardization of cognitive radio systems," IEEE Communications Magazine, vol. 49, no. 3, pp. 82-89, 2011.

[19] B. Zong, C. Fan, X. Wang, X. Duan, B. Wang, and J. Wang, "6G technologies: key drivers, core requirements, system architectures, and enabling technologies," IEEE Vehicular Technology Magazine, vol. 14, no. 3, pp. 18-27, 2019.

[20] S. Dang, O. Amin, B. Shihada, and M. S. Alouini, "What should 6G be?," Nature Electronics, vol. 3, no. 1, pp. 20-29, 2020.

[21] M. Z. Chowdhury, M. Shahjalal, S. Ahmed, and Y. M. Jang, " $6 \mathrm{G}$ wireless communication systems: applications, requirements, technologies, challenges, and research directions," IEEE Open Journal of the Communications Society, vol. 1, pp. 957-975, 2020.

[22] M. M. Aslam, M. N. Irshad, H. Azeem, and M. Ikram, "Cost effective \& energy efficient intelligent smart home system based on IoT," Afyon Kocatepe Üniversitesi Uluslararasi Mühendislik Teknolojileri ve Uygulamal Bilimler Dergisi, vol. 3, no. 1, pp. 10-20, 2020.

[23] A. U. Khan, G. Abbas, Z. H. Abbas, M. Tanveer, S. Ullah, and A. Naushad, "HBLP: a hybrid underlay-interweave mode CRN for the future 5G-based internet of things," IEEE Access, vol. 8, pp. 63403-63420, 2020.

[24] A. Nasser, A. Mansour, and K. C. Yao, "Simultaneous transmitting-receiving-sensing for OFDM-based full-duplex cognitive radio," Physical Communication, vol. 39, article 100987, 2020.

[25] K. E. Lee, J. G. Park, and S. J. Yoo, "Intelligent cognitive radio ad-hoc network: planning, learning and dynamic configuration," Electronics, vol. 10, no. 3, p. 254, 2021.

[26] A. Nasser, H. al Haj Hassan, J. Abou Chaaya, A. Mansour, and K. C. Yao, "Spectrum sensing for cognitive radio: recent advances and future challenge," Sensors, vol. 21, no. 7, pp. 2408-2429, 2021.

[27] F. Qamar, M. U. A. Siddiqui, M. H. D. N. Hindia, R. Hassan, and Q. N. Nguyen, "Issues, challenges, and research trends in spectrum management: a comprehensive overview and new vision for designing $6 \mathrm{~g}$ networks," Electronics, vol. 9, no. 9, pp. 1416-1439, 2020.

[28] B. Barakat, A. Taha, R. Samson et al., "6G opportunities arising from internet of things use cases: a review paper," Future Internet, vol. 13, no. 6, p. 159, 2021.

[29] H. Bin Ahmad, "Ensemble classifier based spectrum sensing in cognitive radio networks," Wireless Communications and Mobile Computing, vol. 2019, Article ID 9250562, 16 pages, 2019.

[30] F. Li, K. Y. Lam, X. Li, Z. Sheng, J. Hua, and L. Wang, "Advances and emerging challenges in cognitive internetof-things," IEEE Transactions on Industrial Informatics, vol. 16, no. 8, pp. 5489-5496, 2020.

[31] X. Yan, H. Xiao, K. An, G. Zheng, and S. Chatzinotas, "Ergodic capacity of NOMA-based uplink satellite networks with randomly deployed users," IEEE Systems Journal, vol. 14, no. 3, pp. 3343-3350, 2020.

[32] S. J. Nawaz, S. K. Sharma, S. Wyne, M. N. Patwary, and M. Asaduzzaman, "Quantum machine learning for $6 \mathrm{G}$ communication networks: state-of-the-art and vision for the future," IEEE Access, vol. 7, no. Ml, pp. 46317-46350, 2019.

[33] J. Jiao, Y. Sun, S. Wu, Y. Wang, and Q. Zhang, "Network utility maximization resource allocation for NOMA in satellitebased internet of things," IEEE Internet of Things Journal, vol. 7, no. 4, pp. 3230-3242, 2020.

[34] Y. C. Liang, D. Niyato, E. G. Larsson, and P. Popovski, “Guest editorial: 6G mobile networks: emerging technologies and applications," China Communications, vol. 17, no. 9, pp. 9091, 2020

[35] R. Alghamdi, R. Alhadrami, D. Alhothali et al., "Intelligent surfaces for $6 \mathrm{G}$ wireless networks: a survey of optimization and performance analysis techniques," IEEE Access, vol. 8, pp. 202795-202818, 2020.

[36] M. M. Aslam, J. Zhang, B. Qureshi, and Z. Ahmed, "Beyond6G- consensus traffic management in CRN , applications, architecture and key challenges," in 2021 IEEE 11th International Conference on Electronics Information and Emergency Communication (ICEIEC) 2021 IEEE 11th International Conference on Electronics Information and Emergency Communication (ICEIEC), pp. 182-185, Beijing, China, 2021.

[37] International Telecommunications Union, IMT Traffic Estimates for the Years 2020 to 2030, Electronic publishing, Geneva, 2015.

[38] K. David and H. Berndt, "6G vision and requirements: is there any need for beyond $5 \mathrm{~g}$ ?," IEEE Vehicular Technology Magazine, vol. 13, no. 3, pp. 72-80, 2018.

[39] 5G IA, European Vision for the 6G Network Ecosystem, 2021.

[40] I. F. Akyildiz, A. Kak, and S. Nie, "6G and beyond: the future of wireless communications systems," IEEE Access, vol. 8, pp. 133995-134030, 2020.

[41] Q. Zhang, A. B. J. Kokkeler, and G. J. M. Smit, Cognitive Radio for Emergency Networks, Mobile Multimedia: Communication Engineering Perspective, 2006.

[42] Y. E. Sagduyu, Y. Shi, A. B. Mackenzie, and Y. T. Hou, "Regret minimization for primary/secondary access to satellite resources with cognitive interference," IEEE Transactions 
on Wireless Communications, vol. 17, no. 5, pp. 3512-3523, 2018.

[43] D. H. M. Shikh-Bahaei, Y.-S. Choi, and D. Hong, "Fullduplex and cognitive radio networking for the emerging $5 \mathrm{G}$ systems," Wireless Communications and Mobile Computing, vol. 2018, Article ID 8752749, 2 pages, 2018.

[44] Z. Ahmed, M. A. Saeed, A. Jenabzadeh, and Z. Weidong, "Frequency domain analysis of resilient consensus in multiagent systems subject to an integrity attack," ISA Transactions, vol. 111, pp. 156-170, 2020.

[45] X. Liu, K.-Y. Lam, F. Li, J. Zhao, and L. Wang, "Spectrum sharing for $6 \mathrm{G}$ integrated satellite-terrestrial communication networks based on NOMA and cognitive radio," 2021, http:// arxiv.org/abs/2101.11418.

[46] Z. Xie, G. Huang, R. Zarei, Z. Ji, H. Ye, and J. He, “A novel nest-based scheduling method for mobile wireless body area networks," Digital Communications and Networks, vol. 6, no. 4, pp. 514-523, 2020.

[47] Y. Zhou, L. Liu, L. Wang et al., "Service-aware 6G: an intelligent and open network based on the convergence of communication, computing and caching," Digital Communications and Networks, vol. 6, no. 3, pp. 253-260, 2020.

[48] M. M. Aslam, M. N. Irshad, H. Azeem, and M. Ikram, "Cost effective \& energy efficient intelligent smart home system based on IoT," Afyon Kocatepe University International Journal of Engineering Technology and Applied Sciences, vol. 3, no. 1, pp. 10-20, 2020.

[49] M. Nasir, M. F. Hayat, A. Jamal, and Z. Ahmed, "Frequency domain consensus control analysis of the networked multiagent system with controller area network bus-induced delay," Journal of Vibration and Control, 2021.

[50] F. Geng, Q. Liu, and P. Zhang, "A time-aware query-focused summarization of an evolving microblogging stream via sentence extraction," Digital Communications and Networks, vol. 6, no. 3, pp. 389-397, 2020.

[51] Y. Wei, M. Peng, and Y. Liu, "Intent-based networks for 6G: insights and challenges," Digital Communications and Networks, vol. 6, no. 3, pp. 270-280, 2020.

[52] P. Zhang and X. Kang, "Similar physical entity matching strategy for mobile edge search," Digital Communications and Networks, vol. 6, no. 2, pp. 203-209, 2020.

[53] S. Rani, A. Kataria, V. Sharma et al., "Threats and corrective measures for IoT security with observance of cybercrime: a survey," Wireless Communications and Mobile Computing, vol. 2021, Article ID 5579148, 30 pages, 2021.

[54] Y. H. Robinson, T. S. Lawrence, E. G. Julie, and S. Vimal, "Development of fuzzy enabled coverage hole detection algorithm in wireless sensor networks," Wireless Personal Communications, vol. 119, no. 4, pp. 3631-3649, 2021.

[55] F. Dressler and F. Kargl, "Towards security in nano-communication: challenges and opportunities," Nano Communication Networks, vol. 3, no. 3, pp. 151-160, 2012.

[56] L. Mucchi, A. Martinelli, S. Jayousi, S. Caputo, and M. Pierobon, "Secrecy capacity and secure distance for diffusion-based molecular communication systems," IEEE Access, vol. 7, pp. 110687-110697, 2019.

[57] J. Walko, “Cognitive radio," IEE Review, vol. 51, no. 5, pp. 3437, 2005.

[58] J. Mitola and G. Q. Maguire, "Cognitive radio: making software radios more personal," IEEE Personal Communications, vol. 6 , no. 4 , pp. 13-18, 1999.
[59] S. Vimal, Y. H. Robinson, M. Kaliappan, K. Vijayalakshmi, and S. Seo, "A method of progression detection for glaucoma using K-means and the GLCM algorithm toward smart medical prediction," The Journal of Supercomputing, vol. 77, no. 10, pp. 11894-11910, 2021.

[60] X. Ling, J. Wang, T. Bouchoucha, B. C. Levy, and Z. Ding, "Blockchain radio access network (B-RAN): towards decentralized secure radio access paradigm," IEEE Access, vol. 7, no. c, pp. 9714-9723, 2019.

[61] K. Kotobi and S. G. Bilen, "Secure blockchains for dynamic spectrum access: a decentralized database in moving cognitive radio networks enhances security and user access," IEEE Vehicular Technology Magazine, vol. 13, no. 1, pp. 32-39, 2018.

[62] J. Ma, R. Shrestha, J. Adelberg et al., "Security and eavesdropping in terahertz wireless links," Nature, vol. 563, no. 7729, pp. 89-93, 2018.

[63] I. F. Akyildiz, J. M. Jornet, and C. Han, "Terahertz band: next frontier for wireless communications," Physical Communication, vol. 12, pp. 16-32, 2014.

[64] M. H. Alsharif, M. A. M. Albreem, A. A. A. Solyman, and S. Kim, "Toward $6 \mathrm{~g}$ communication networks: terahertz frequency challenges and open research issues," Computers, Materials \& Continua, vol. 66, no. 3, pp. 2831-2842, 2021.

[65] P. Singh, M. Khari, and S. Vimal, "EESSMT: an energy efficient hybrid scheme for securing mobile ad hoc networks using IoT," in Wireless Personal Communications, Springer, 2021.

[66] M. A. Adedoyin and O. E. Falowo, "Combination of ultradense networks and other $5 \mathrm{G}$ enabling technologies: a survey," IEEE Access, vol. 8, pp. 22893-22932, 2020.

[67] F. Nawaz, J. Ibrahim, M. Awais, M. Junaid, S. Kousar, and T. Parveen, "A review of vision and challenges of $6 \mathrm{G}$ technology," International Journal of Advanced Computer Science and Applications, vol. 11, no. 2, pp. 643-649, 2020.

[68] R. A. Stoica and G. T. F. de Abreu, "6G: the wireless communications network for collaborative and AI applications," 2019, https://arxiv.org/abs/1904.03413.

[69] J. Zhao, "A survey of intelligent reflecting surfaces (IRSs): towards $6 \mathrm{G}$ wireless communication networks with massive MIMO 2.0," 2019, https://arxiv.org/abs/1907.04789.

[70] S. Ali, W. Saad, N. Rajatheva et al., "6G white paper on machine learning in wireless communication networks," 2020, https://arxiv.org/abs/2004.13875.

[71] M. Cesana, F. Cuomo, and E. Ekici, "Routing in cognitive radio networks: challenges and solutions," Ad Hoc Networks, vol. 9, no. 3, pp. 228-248, 2011.

[72] M. H. Alsharif, A. H. Kelechi, M. A. Albreem, S. A. Chaudhry, M. S. Zia, and S. Kim, "Sixth generation (6G) wireless networks: vision, research activities, challenges and potential solutions," Symmetry, vol. 12, no. 4, p. 676, 2020.

[73] K. Tekbıyık, A. R. Ekti, G. K. Kurt, and A. Görçin, "Terahertz band communication systems: challenges, novelties and standardization efforts," Physical Communication, vol. 35, article 100700, 2019.

[74] S. Bashir, M. H. Alsharif, I. Khan et al., "Mimo-terahertz in $6 \mathrm{~g}$ nano-communications: channel modeling and analysis," Computers, Materials and Continua, vol. 66, no. 1, pp. 263274, 2021.

[75] W. Chen, X. Yang, S. Jin, and P. Xu, "Sparse array of sub-surface aided blockage-free multi-user mmWave communication 
systems," Digital Communications and Networks, vol. 6, no. 3, pp. 292-303, 2020.

[76] I. A. Khoso, T. B. Javed, S. Tu et al., "A fast-convergent detector based on joint jacobi and richardson method for uplink massive MIMO Systems," in 2019 28th Wireless and Optical Communications Conference (WOCC), Beijing, China, 2019.

[77] M. Akhlaq, H. Farooq, and Z. Ahmad, "Performance analysis of X-band RADAR in the presence of electronic jammers," in 2019 International Conference on Electrical, Communication, and Computer Engineering (ICECCE). IEEE, pp. 1-4, Swat, Pakistan, July 2019.

[78] M. Attarifar, A. Abbasfar, and A. Lozano, "Modified conjugate beamforming for cell-free massive MIMO," IEEE Wireless Communications Letters, vol. 8, no. 2, pp. 616-619, 2019.

[79] I. A. Khoso, X. Dai, M. N. Irshad, A. Khan, and X. Wang, "A low-complexity data detection algorithm for massive MIMO systems," IEEE Access, vol. 7, pp. 39341-39351, 2019.

[80] Z. Gu, J. Zhang, Y. Ji, L. Bai, and X. Sun, "Network topology reconfiguration for FSO-based fronthaul/backhaul in 5G+ wireless networks," IEEE Access, vol. 6, no. c, pp. 69426-69437, 2018.

[81] A. Douik, H. Dahrouj, T. Y. Al-Naffouri, and M. S. Alouini, "Hybrid radio/free-space optical design for next generation backhaul systems," IEEE Transactions on Communications, vol. 64, no. 6, pp. 2563-2577, 2016.

[82] H. Yu, M. K. Afzal, Y. B. Zikria, A. Rachedi, and F. H. P. Fitzek, "Tactile internet: technologies, test platforms, trials, and applications," Future Generation Computer Systems, vol. 106, pp. $685-688,2020$.

[83] M. S. Miah, K. M. Ahmed, M. K. Islam, M. A. R. Mahmud, M. M. Rahman, and H. Yu, "Enhanced sensing and sumrate analysis in a cognitive radio-based internet of things," Sensors (Switzerland), vol. 20, no. 9, p. 2525, 2020.

[84] A. Ostovar, Y. Bin Zikria, H. S. Kim, and R. Ali, "Optimization of resource allocation model with energy-efficient cooperative sensing in green cognitive radio networks," IEEE Access, vol. 8, pp. 141594-141610, 2020.

[85] M. A. Naeem, R. Ali, M. Alazab, M. Yhui, and Y. Bin Zikria, "Enabling the content dissemination through caching in the state-of-the-art sustainable information and communication technologies," Sustainable Cities and Society, vol. 61, article 102291, 2020.

[86] H. Xu, H. Gao, C. Zhou, R. Duan, and X. Zhou, "Resource allocation in cognitive radio wireless sensor networks with energy harvesting," Sensors, vol. 19, no. 23, 2019.

[87] F. Al-Turjman, E. Ever, Y. Bin Zikria, S. W. Kim, and A. Elmahgoubi, "SAHCI: scheduling approach for heterogeneous content-centric IoT applications," IEEE Access, vol. 7, pp. 80342-80349, 2019.

[88] S. Lee, J. Youn, and B. C. Jung, “A cooperative phase-steering technique with on-off power control for spectrum sharingbased wireless sensor networks," Sensors, vol. 20, no. 7, 2020.

[89] B. S. Muwonge, T. Pei, J. S. Otim, and F. Mayambala, "A joint power, delay and rate optimization model for secondary users in cognitive radio sensor networks," Sensors (Switzerland), vol. 20, no. 17, pp. 4907-4918, 2020.

[90] H. A. Bany Salameh, M. M. Krunz, and O. Younis, "MAC protocol for opportunistic cognitive radio networks with soft guarantees," IEEE Transactions on Mobile Computing, vol. 8, no. 10, pp. 1339-1352, 2009.

[91] Y. Wang, G. Zheng, H. Ma, Y. Li, and J. Li, "A joint channel selection and routing protocol for cognitive radio network,"
Wireless Communications and Mobile Computing, vol. 2018, Article ID 6848641, 7 pages, 2018.

[92] H. S. Ghazi and K. Wesołowski, "Application of an interference cancellation detector in a two-way relaying system with physical network coding," Electron, vol. 10, no. 11, p. 1294, 2021.

[93] F. Brunero, D. Tuninetti, and N. Devroye, "On code design for wireless channels with additive radar interference," in 2019 IEEE Information Theory Workshop (ITW), pp. 3-7, Visby, Sweden, 2019.

[94] H. Shajaiah, A. Abdelhadi, and C. Clancy, "Resource allocation with carrier aggregation for commercial use of $3.5 \mathrm{GHz}$ spectrum," in Resource Allocation with Carrier Aggregation in Cellular Networks, Springer, Cham, 2018.

[95] A. H. Moon, U. Iqbal, and G. Mohiuddin Bhat, "Authenticated key exchange protocol for wireless sensor networks," International Journal of Applied Engineering Research, vol. 11, no. 6, pp. 4280-4287, 2016.

[96] V. Pla, A. S. Alfa, J. Martinez-Bauset, and V. Casares-Giner, "Discrete-time analysis of cognitive radio networks with nonsaturated source of secondary users," Wireless Communications and Mobile Computing, vol. 2019, Article ID 7367028, 12 pages, 2019.

[97] B. Zayen, A. Hayar, and K. Kansanen, "Blind spectrum sensing for cognitive radio based on signal space dimension estimation," in 2009 IEEE International Conference on Communications, Dresden, Germany, 2009.

[98] Y. Arjoune and N. Kaabouch, "A comprehensive survey on spectrum sensing in cognitive radio networks: recent advances, new challenges, and future research directions," Sensors, vol. 19, no. 1, 2019.

[99] A. Abu Alkheir and H. T. Mouftah, "Cognitive radio for public safety communications," in Wireless Public Safety Networks, Elsevier, 2016.

[100] T. Bräysy, J. Lehtomäki, B. Calvet, S. Delmas, and C. Moy, Cognitive Techniques for Finding Spectrum for Public Safety Services, Oulu Univ (Finland), 2010.

[101] J. Li and B. J. Hu, "Quantized cooperative spectrum sensing in bandwidth- constrained cognitive V2X based on deep learning," Electron, vol. 10, no. 11, p. 1315, 2021.

[102] Y. Cao, E. J. Sunde, and K. Chen, "Multiplying channel capacity: aggregation of fragmented spectral resources," IEEE Microwave Magazine, vol. 20, no. 1, pp. 70-77, 2019.

[103] A. Goldsmith and I. MaríC, Capacity of Cognitive Radio Networks, Cambridge University Press, 2012.

[104] L. F. Minervini, "Spectrum management reform: rethinking practices," Telecommunications Policy, vol. 38, no. 2, pp. 136-146, 2014.

[105] A. S. Alfa, V. Pla, J. Martinez-Bauset, and V. Casares-Giner, "Discrete time analysis of cognitive radio networks with saturated source of secondary users," in NETWORKING 2011 Workshops, vol. 6827 of Lecture Notes in Computer Science, pp. 3-12, Springer, Berlin, Heidelberg, 2011.

[106] M. Hassan, M. Singh, and K. Hamid, "Overview of cognitive radio networks," Journal of Physics Conference Series, vol. 1, p. 2021, 2021.

[107] A. Jagannath, J. Jagannath, and T. Melodia, "Redefining wireless communication for 6G: signal processing meets deep learning," 2020, https://arxiv.org/abs/2004.10715. 